\title{
The fourth circuit element
}

Arising from: D. B. Strukov, G. S. Snider, D. R. Stewart \& R. S. Williams Nature 453, 80-83 (2008)

In 1971, Chua suggested ${ }^{1}$ that there should in principle exist a circuit element linking electrical charge, $q$, and magnetic flux, $\varphi$. Strukov et $a l^{2}$ claim recently to have found such a link. However, here I point out that Chua's suggestion was in fact preceded by experimental evidence $^{3-5}$ for magneto-electric effects whereby magnetic and electrical signals are interconverted. When the stimulus is magnetic and the response is electrical, the linear magneto-electric coupling constant $^{5}, \alpha$, is typically reported as $\mathrm{d} P / \mathrm{d} H$, where $P$ represents the electrical polarization and $H$ represents the applied magnetic field. The coupling constant could equally be presented as $\mathrm{d} q / \mathrm{d} \varphi$ if divided by the permeability of free space.

In making their claim, Strukov et al. therefore overlook the magneto-electric literature. In magneto-electric systems, the link arises explicitly and through a simple geometry, whereas Strukov et al. consider a system in which magnetic signals are neither applied nor measured. Moreover, some magneto-electric systems display nonlinear coupling ${ }^{6,7}$, which is necessary but not sufficient for memristance $^{1,2}$.

Neil D. Mathur ${ }^{1}$

${ }^{1}$ Department of Materials Science, New Museums Site, Pembroke Street, Cambridge CB2 3QZ, UK.

e-mail:ndm12@cam.ac.uk

Received 27 May; accepted 28 August 2008.
1. Chua, L. O. Memristor - the missing circuit element. IEEE Trans. Circuit Theory 18, 507-519 (1971).

2. Strukov, D. B., Snider, G. S., Stewart, D. R. \& Williams, R. S. The missing memristor found. Nature 453, 80-83 (2008).

3. Astrov, D. N. The magnetoelectric effect in antiferromagnetics. Zh. Eksp. Teor. Fiz. 38, 984-985; Sov. Phys. JETP 11, 708-709 (1960).

4. Folen, V. J., Rado, G. T. \& Stalder, E. W. Anisotropy of the magnetoelectric effect in $\mathrm{Cr}_{2} \mathrm{O}_{3}$. Phys. Rev. Lett. 6, 607-608 (1961).

5. Ascher, E., Rieder, H., Schmid, H. \& Stössel, H. Some properties of ferromagnetoelectric nickel-iodine boracite, $\mathrm{Ni}_{3} \mathrm{~B}_{7} \mathrm{O}_{13}$ l. J. Appl. Phys. 37, 1404-1405 (1966).

6. Hou, S. L. \& Bloembergen, N. Paramagnetoelectric effects in $\mathrm{NiSO}_{4} \cdot 6 \mathrm{H}_{2} \mathrm{O}$. Phys. Rev. 138, A1218-A1226 (1965).

7. Scott, J. F. Mechanisms of dielectric anomalies in $\mathrm{BaMnF}_{4}$. Phys. Rev. B 16, 2329-2331 (1977).

doi:10.1038/nature07437 